\title{
Acesso aos serviços de saúde na rede de atenção: compreendendo a narrativa de profissionais de saúde
}

\section{Access to health services in the care network: understanding the narrative of health professionals}

\author{
Rose Ferraz Carmo ${ }^{1,2}$ (D), Débora Noara Duarte dos Santos² (D), Juliana Ferreira de Oliveira² (D), \\ Celina Maria Modena ${ }^{2}$ (D), Josélia Oliveira Araújo Firmo² (D) Zélia Maria Profeta da Luz ${ }^{2}$ (D) \\ 'Escola de Saúde Pública do Estado de Minas Gerais - Belo Horizonte (MG), Brasil. \\ ${ }^{2}$ Fundação Oswaldo Cruz, Instituto René Rachou - Belo Horizonte (MG), Brasil.
}

\begin{abstract}
Como citar: Carmo RF, Santos DND, Oliveira JF, Modena CM, Firmo JOA, Luz ZP. Acesso aos serviços de saúde na rede de atenção: compreendendo a narrativa de profissionais de saúde. Cad Saúde Colet, 2021;29(1):77-85. https://doi.org/10.1590/1414-462X202129010512
\end{abstract}

\section{Resumo}

Introdução: Durante as três décadas do Sistema Único de Saúde (SUS), a tríade universalidade/ integralidade/igualdade esteve complementada pela garantia de acesso, que, apesar de importantes avanços, também registrou a manutenção de desafios e, até, retrocessos. Objetivo: Compreender os aspectos imbricados com o acesso à rede de atenção à saúde. Método: Entrevistas e grupos focais foram realizados com 32 profissionais de saúde, incluindo gestores, de um município da Região Metropolitana de Belo Horizonte caracterizado por marcada vulnerabilidade social e econômica. As dimensões teóricas "estrutural", "operacional" e "relacional" guiaram a análise e interpretação da narrativa dos profissionais. Resultados: Os resultados demonstraram que a dimensão estrutural assumiu centralidade na narrativa dos profissionais de saúde como determinantes para o acesso, relacionada não apenas ao financiamento, mas também a questões afetas à operacionalização e organização dos serviços de saúde, como rotatividade dos profissionais e planejamento territorial. Conclusão: Muitas dessas questões não são passíveis de intervenções, pelo menos de forma direta e em curto prazo pelos profissionais de saúde, incluindo gestores. No entanto, reconhecer e refletir sobre sua influência no acesso aos serviços da rede de saúde municipal pode ser um produtivo caminho no sentido de articulação, proposição e implementação de estratégias. Palavras-chave: acesso a serviços de saúde; política de saúde; cuidados de saúde.

\begin{abstract}
Background: During the three decades of the Unified Health System (SUS), the triad of universality/ integrality/equality was complemented by the guarantee of access, which despite important advances, also registered the maintenance of challenges and even setbacks. Objective: To understand the factors that influenced access to public health services. Method: Interviews and focus groups were realized with 32 health professionals including managers from a municipality in the metropolitan region of Belo Horizonte characterized by the marked social and economic vulnerability. The theoretical structural, operational, and relational dimensions guided the analysis and interpretation of the professionals' narrative. Results: The results showed that the structural dimension assumed a central role in the narrative of health professionals as determinants of access related not only to financing but also to questions related to the operability and organization of health services such as professional rotation and territorial planning. Conclusion: Many of these issues are not amenable to interventions at least directly and in short terms by health professionals including managers, however, recognizing and reflecting on their
\end{abstract}


influence on access to municipal health network services can be a productive path towards articulation, proposition, and implementation's strategies.

Keywords: health services access; health policy; health care.

\section{INTRODUÇÃO}

Revisitar alguns aspectos da trajetória do Sistema Único de Saúde (SUS) tem, no ano em que comemoramos seu aniversário de 30 anos, um significado singular, já que o sistema completa três décadas em um contexto político que ameaça sua manutenção. Em todo seu percurso, a tríade universalidade/integralidade/igualdade esteve complementada pela garantia de acesso ', que registrou importantes mudanças, incluindo ampliação da oferta de serviços e profissionais vinculados ao SUS ${ }^{2}$. Como ressaltam Viacava et al. ${ }^{3}$, indicadores comumente usados na avaliação do acesso aos serviços de saúde, como o percentual de pessoas que referem ter consultado médico e dentista nos últimos 12 meses, apontam que mais pessoas têm procurado e obtido serviços de saúde. No entanto, como destacam os autores, desafios históricos, incluindo a relação público-privado na prestação dos serviços de saúde, as marcantes desigualdades regionais e o subfinanciamento contribuem para manutenção de dificuldades, problemas e, até, retrocessos ${ }^{2,3}$.

Buscando refletir sobre os fatores que influenciam o acesso aos serviços de saúde, retomamos um estudo realizado, em 2011 por nossa equipe de pesquisa em um município da Região Metropolitana de Belo Horizonte caracterizado por marcada vulnerabilidade social ${ }^{4}$. Partindo do pressuposto de que desigualdades sociais no acesso aos serviços de saúde, assim como nas condições de saúde e vida da população expressam oportunidades diferenciadas em função da posição social do indivíduo e caracterizam situações de injustiça social ${ }^{5}$, o objetivo do estudo realizado em 2011 foi identificar fatores que limitavam o acesso aos serviços públicos de saúde a partir da perspectiva de profissionais de saúde e usuários de uma Unidade Básica de Referência (UBR).

Os resultados do estudo realizado em 2011 evidenciaram fatores determinantes para o acesso do grupo estudado, incluindo a aceitabilidade do sistema de saúde pelos usuários, a relação entre estes e os profissionais de saúde, a adequação do número de profissionais para a prestação de serviços, a atenção secundária/referência, o financiamento do sistema de saúde e a estigmatização do município 4 .

Considerando que protagonizar o nível local (município) — menor unidade administrativa - de investigação permite acessar especificidades e particularidades de uma realidade que importa ao entendimento das redes de atenção à saúde, já que corresponde ao maior nível de desagregação da gestão e execução de ações, ampliamos nosso território de análise de uma UBR, como no estudo anterior, para a rede de serviços públicos de saúde do município.

Nosso objetivo no estudo atual foi identificar fatores que influenciavam o acesso aos serviços públicos de saúde e identificar possíveis estratégias prioritárias de atuação.

\section{MÉTODO}

O estudo foi desenvolvido entre 2015 e 2017 em um município da Região Metropolitana de Belo Horizonte, que ocupa uma área territorial de $155,5 \mathrm{Km} 2$, onde vive uma população estimada de aproximadamente 330.000 habitantes $^{6}$.

O município possuía, em 2010, Índice de Desenvolvimento Humano (IDH) igual a 0,684 e Índice de Vulnerabilidade Social (IVS) de 0,3587 , ambos índices considerados médios segundo as respectivas escalas.

Do ponto de vista da territorialização para a gestão da saúde municipal, o município é dividido em cinco Regiões Sanitárias (RS). No período do estudo, conforme dados disponibilizados pela Secretaria Municipal de Saúde (SMS), a rede de serviços de assistência à saúde estava distribuída nas cinco RS, e contava com 50 UBS, cinco UBR e nove unidades de Atenção Ambulatorial Especializada.

Na Atenção Primária, existiam 53 Equipes de Saúde da Família (ESF) alocadas em 50 UBS cobrindo $50 \%$ da população, além de cinco UBR à saúde, sendo uma por Região Sanitária e 
que não tinham ESF. Em todas as RS havia um profissional responsável pelo acompanhamento das ESF e um profissional responsável pelas UBR.

Na Atenção Secundária, o município dispunha de rede própria de Atenção Ambulatorial Especializada, ofertando serviços de média e alta complexidade em nove unidades próprias: Centro de Especialidades Médicas e Odontológicas, Ambulatório de Referência em Oftalmologia, Ambulatório de Referências para Doenças Infectocontagiosas e Parasitárias, Clínica de Reabilitação, Centro Viva Vida de Referência Secundária, Centro de Especialidades Odontológicas e três Unidades Ambulatoriais de Saúde Mental.

Essa rede de serviços especializados também contava com apoio do hospital municipal, onde eram realizados alguns serviços e exames de diagnóstico por imagem. O hospital, do tipo geral e de médio porte, ofertava atendimento total pelo SUS, sendo porta aberta em urgência/ emergência e de internação de média complexidade em clínica médica, pediátrica, cirúrgica, obstétrica e traumato-ortopédica.

O hospital contava com uma capacidade instalada de 107 leitos assim distribuídos: 14 leitos para cirurgia; 18 leitos para obstetrícia; 49 leitos para clínica geral; 18 leitos para pediatria (cinco para o berçário); dois leitos para ortopedia; um leito para neurologia; um leito para AIDS e quatro leitos para diagnóstico terapêutico.

\section{Procedimentos metodológicos}

Os pressupostos teóricos metodológicos da Pesquisa Qualitativa em Saúde ${ }^{8}$ nortearam o estudo. Para mapear e compreender o quadro empírico envolvido com o acesso aos serviços de saúde nesse território, o estudo incluiu gestores e profissionais dos diferentes níveis de atenção à saúde abarcando, portanto, diferentes formas de vinculação/relação com o tema a ser investigado.

Os instrumentos de coleta de informações utilizados foram o grupo focal e a entrevista semiestruturada. Em ambos, a fala assume centralidade pela possibilidade de conter a experiência, de revelar condições estruturais, sistemas de valores, normas e símbolos e de permitir o entendimento intersubjetivo ${ }^{8}$.

Os grupos focais foram realizados com supervisores da APS do município e com ESF de Unidades Básicas de Saúde (UBS) localizadas em cada uma das Regiões Sanitárias do município e com a equipe de profissionais das Unidades Básicas de Referência (UBR). As entrevistas foram realizadas com profissionais que atuavam em unidades de saúde da média complexidade ambulatorial e do hospital do município.

O número de grupos focais e entrevistas foi dimensionado pelo critério de saturação teórica9,10. Desta forma, a coleta de informações foi encerrada quando constatamos que a inclusão de novos indivíduos não forneceria novos elementos para aumentar a abrangência e aprofundar a compreensão do nosso objeto de estudo.

Ambos, entrevistas e grupos focais, foram guiados por um roteiro elaborado previamente que, mantendo as especificidades relativas aos profissionais de cada nível de atenção, abordavam características do atendimento local, da equipe de saúde, dos usuários e da rede prestadora de serviços, conforme descrito no Quadro 1.

Todos os depoimentos foram gravados, integralmente transcritos e tratados pela Análise de Conteúdo ${ }^{11}$. Os grupos focais e entrevistas foram realizados somente após a assinatura do Termo de Consentimento Livre e Esclarecido e o estudo foi aprovado pelo Comitê de Ética em Pesquisa do Instituo René Rachou (CAAE 11350413.0.0000.5091).

Os depoimentos foram identificados, nos resultados, de acordo com o nível de atenção à saúde: Atenção Primária à Saúde (APS), Atenção Secundária à Saúde (AS) e Atenção Terciária à Saúde (AT).

\section{Análise dos dados}

A sistematização teórica desenvolvida no estudo de Viegas et al. ${ }^{4}$ foi utilizada para compreender o acesso à rede de atenção à saúde. A dimensão "estrutural", mais distal, corresponde a fatores como contexto socioeconômico do município que impacta não apenas 
Quadro 1. Dimensões do acesso aos serviços de saúde abordadas no roteiro utilizado nas entrevistas e grupos focais e respectivas variáveis analisadas

\begin{tabular}{|l|l|}
\hline \multicolumn{1}{|c|}{ Dimensões do acesso } & \multicolumn{1}{c|}{ Variáveis } \\
\hline Acessibilidade sócio-organizacional & $\begin{array}{l}\text { Horário de funcionamento; dinâmica de marcação de consultas; } \\
\text { tempo de espera }\end{array}$ \\
\hline Acessibilidade geográfica & $\begin{array}{l}\text { Meio de transporte utilizado pelos usuários; tempo de } \\
\text { deslocamento da residência à Unidade de Saúde }\end{array}$ \\
\hline Disponibilidade & Demandas existentes; demandas atendidas \\
\hline Acessibilidade econômica & $\begin{array}{l}\text { Relacionadas à impossibilidade de acesso devido a questões } \\
\text { econômicas }\end{array}$ \\
\hline Aceitabilidade & Relação usuário x profissional; relação usuário x serviço de saúde \\
\hline Fatores intervenientes & Fatores facilitadores e dificultadores \\
\hline Possíveis caminhos & $\begin{array}{l}\text { Possibilidades de intervenção para melhorias no acesso aos } \\
\text { serviços de saúde }\end{array}$ \\
\hline
\end{tabular}

o acesso, mas, de forma mais abrangente, as condições de vida da população. A dimensão "operacional" ocupa uma posição intermediária e esteve, na fala dos participantes do estudo, relacionada à organização dos serviços de saúde; e a dimensão "relacional" abarca uma gama de aspectos subjetivos envolvidos na relação entre usuários e profissionais de saúde.

\section{RESULTADOSE DISCUSSÃO}

Foram realizados sete grupos focais e seis entrevistas. O primeiro grupo focal foi realizado com supervisores da APS. Participaram quatro supervisoras - incluindo a responsável pela UBR do município - e um supervisor. Todos tinham idade variando entre 26 e 32 anos, eram enfermeiras(os) graduadas(os) com tempo de atuação no cargo variando entre 5 e 24 meses.

Os demais grupos focais foram realizados com profissionais da ESF de uma UBS de cada uma das cinco Regiões Sanitárias do município e com a equipe da UBR municipal. Entre esses profissionais, 26 (89,6\%) eram do sexo feminino, com idade variando de 27 a 62 anos e 8 (27,6\%) tinham nível superior de formação. $O$ tempo de atuação no cargo variou de 1 a 14 anos.

As entrevistas foram realizadas em unidades de saúde de média complexidade ambulatorial do município com profissionais atuantes nesses serviços, incluindo duas enfermeiras, uma psicóloga, uma dentista e uma gestora de serviços de saúde. A idade das participantes variou entre 26 e 50 anos e o tempo de atuação no cargo entre 6 meses e 7 anos.

$\mathrm{Na}$ Atenção Terciária foi entrevistada a coordenadora do hospital do município, que atuava no cargo a quatro anos e tinha formação em enfermagem.

\section{Fatores relacionados ao acesso aos serviços de saúde}

As dimensões teóricas "estrutural", "operacional" e "relacional" em articulação com as narrativas dos entrevistados revelaram que, assim como no estudo desenvolvido no município em 2011, o financiamento do sistema de saúde, a organização dos serviços e a relação estabelecida entre profissionais e usuários influenciavam o acesso aos serviços de saúde.

A questão da vulnerabilidade econômica do município foi relatada pelos profissionais: "Acho que esse fator econômico não é da população não, é do município" (APS); "[...] falta mesmo muito investimento, assim, o posto de saúde, esses equipamentos estão todos, né, com defeito. Desde que nós entramos tá da mesma forma. Eu tenho seis anos que estou aqui [...]" (APS); "[...] o que tá faltando na, nas unidades ultimamente nunca faltou [...] mas tá faltando, assim, remédio, medicação, o básico, álcool, é, álcool pra fazer higienização" (APS). Em relação ao financiamento é importante considerar que, em $2015,74,8 \%$ do orçamento municipal era proveniente de fontes externas e apenas $9,8 \%$ da população tinham ocupação formal ${ }^{7}$, refletindo, indubitavelmente, na 
arrecadação de impostos. No entanto, é importante considerar que a questão do financiamento constitui um desafio estrutural não apenas para o serviço de saúde do município estudado, mas para o Brasil como um todo, onde $54 \%$ do gasto em saúde acontecem no setor privado responsável pelo atendimento de apenas $25 \%$ da população ${ }^{2}$. Com insuficientes recursos, o SUS enfrenta problemas na manutenção da rede de serviços e na remuneração de seus trabalhadores, limitando a democratização do acesso, o que, vale lembrar, sempre esteve em disputa em função dos interesses de governos nas várias esferas administrativas ${ }^{3,12,13}$.

A dimensão operacional abarcou, na fala dos participantes, uma gama de aspectos relacionados à organização dos serviços de saúde. Profissionais e gestores relataram aspectos específicos do nível de atenção em que estavam inseridos, como a rotatividade de profissionais de saúde na APS "[...] se o profissional sabe que vai ficar aqui só um ano, ele não tem comprometimento, e a atenção básica é comprometimento. Você tem que conhecer o paciente, você não conhece a doença, você conhece o paciente, e o paciente você só conhece com o tempo" (APS), como também lacunas no acesso integral à rede de serviços de saúde município "[...] as pessoas não têm acesso a todos os níveis [...] piorou a média, porque, no caso, tem muito encaminhamento pras especialidades e a gente não tem vaga..." (APS).

A rotatividade de profissionais, sobretudo na APS, é vivenciada em outros territórios brasileiros. Medeiros et al. ${ }^{13}$ problematizaram a questão ao estudar os fatores que influenciavam a rotatividade de médicos e enfermeiros da Estratégia Saúde da Família no Vale do Taquari, Rio Grande do Sul. Fatores como precarização do vínculo de trabalho, fragmentação da formação, ausência de vínculo com a comunidade e más condições de trabalho foram relatados pelos participantes do estudo. Esses fatores foram, em certa medida, abordados por Machado e Ximenes ${ }^{14}$, em artigo recente, onde as autoras fazem uma reflexão sobre a Política de Gestão do Trabalho e da Educação na Saúde nas três décadas de SUS. As autoras enfatizam o entendimento de que os trabalhadores da saúde, assim como a saúde, constituem um bem público, o que pressupõe a garantia de requisitos básicos para a valorização do trabalhador e do seu trabalho: plano de cargos, carreiras e salários; vínculos de trabalho com proteção social; espaços de discussão e negociação do trabalho; educação permanente; humanização da qualidade do trabalho, dentre outros ${ }^{14}$.

Em um município que convive com marcada vulnerabilidade social e econômica e ainda com estigma de abrigar cinco presídios, as questões pontuadas acima assumem uma conotação ainda mais problemática, já que é permeada por fatores contextuais, a exemplo da violência que cerceia a atuação e manutenção dos profissionais de saúde, "[...] porque às vezes o PSF a gente abria, mas era meio com medo, entendeu? Tinha muita violência; [...]. Tem a questão também da segurança da unidade, aqui não tem nenhuma." (APS).

No que diz respeito à fragilidade no acesso integral aos serviços de saúde, relatada pelos participantes do estudo, é importante considerar que no Brasil a organização da assistência é operada por Regiões e Redes de Atenção à Saúde. Viana et al. ${ }^{1}$ discutem que um dos desafios centrais para consolidação desse tipo de organização é a incorporação do sentido ético, com centralidade nas necessidades sociais, populacionais e individuais, derivadas da integralidade, dos direitos humanos e sociais, em amplas interações multiescalares e intersetoriais. Os autores sinalizam apoiados em Paim ${ }^{15: 571}$ a incompatibilidade de um modelo único de acesso à APS "[...] dada a heterogeneidade das situações que compõem a realidade de saúde nas diferentes regiões, cidades e territórios do país".

A esse respeito é importante considerar, subjacente ao processo de construção das Regiões e Redes de Atenção à Saúde, a territorialização em saúde que adquire contornos de política por constituir uma proposta do Estado para a organização da APS em todo país, mas é também uma técnica de planejamento utilizada para se delimitar as áreas de atuação das unidades de atenção primária à saúde. Apesar de considerar diferentes realidades, como aquelas vivenciadas por populações ribeirinhas e moradores de rua, a Política Nacional de Atenção Básica (PNAB) ainda condiciona numericamente as ESF a uma oferta e a um teto financeiro, sendo necessária a definição, a priori, de uma população a ser atendida ${ }^{16}$.

Esse "modelo" de acesso em APS, de acordo com Viana et al. ${ }^{1}$ encontra limites históricos ao definir pelo paciente o tipo de acesso e ofertas de serviços. Para Pereira e Barcellos ${ }^{17}$, o 
planejamento territorial, baseado exclusivamente na quantificação da população para a definição de recortes territoriais sem a incorporação de nenhuma proposta de tipificação dos territórios, limita a eficácia das ações.

Ainda que os serviços de saúde inseridos na APS operem mais fortemente sob a lógica da territorialização, pois são organizados em uma base territorial bem definida, a organização desse nível de atenção repercute nos demais, podendo comprometer o ordenamento do acesso ${ }^{18}$, o que fica claro na fala de uma participante do estudo: "[...] nosso problema ainda é a atenção primária que não funciona como deveria funcionar, porque eles demoram... visando o ponto de vista do paciente, é aquele negócio, né, no hospital sempre tem médico, então, é lá que eu vou conseguir". Ademais, por representar o papel de porta de entrada e ordenadora de todo o sistema, a APS precisa estar organizada territorialmente de forma a potencializar a Saúde da Família, sua estratégia prioritária ${ }^{19}$.

Na prática, a conformação dos territórios de atuação das unidades de saúde impõe uma lógica de funcionamento que pode restringir o acesso:

[...] PSF tem aquela questão de, ah, não pode, porque passou de tantas mil pessoas, não interessa se aquele paciente more do lado do posto, interessa é se eu, eu tenho mais de 4 mil pessoas, né, tô citando, não sei nem se é 4 mil, mas se eu passar de 4 mil é aí, tá no programa 4 mil, ele éo 4.001 , eu moro do lado do posto e eu não posso ser atendido lá, porque o programa não me aceita mais, e aí eu venho pra cá e aqui a distância eu não sei falar em quilômetros, mas eu sei que é bem longe, sabe? (APS).

Faria e Bortolozzi $i^{2019}$ destacam que a criação de um limite territorial novo, definido como limite de atuação dos serviços de saúde, pode não representar o território de vida das pessoas, pois "[...] o território já existe como totalidade, não se cria outro território ou limite [...]". Para contemplar o território como totalidade, Faria ${ }^{21}$ e Gondim et al. ${ }^{22}$ sugerem, apoiados na produção de Milton Santos ${ }^{23,24}$, que seria fundamental (re)conhecer o território e seus contextos de uso.

Segundo os autores, esse caminho permitiria inverter a lógica da territorialização em saúde baseada na alocação de serviços, definição de público-alvo e delimitação do território, abarcando práticas sociais e modos de viver a vida que importam ao planejamento de práticas inerentes às políticas governamentais como alocação efetiva dos serviços de saúde.

É importante considerar ainda que o limite, em territórios onde não há universalização dos serviços de saúde, limita duplamente, incluindo de um lado e excluindo de outro ${ }^{21}$. Esse é o cenário do estudo, com cobertura de $50 \%$ da população pela Estratégia Saúde da Família, o serviço de saúde não absorve a demanda, o que gera exclusão e iniquidades: "[...] a demanda é muito grande, né [...] com a dificuldade de profissionais né, que a gente observa aí, de consultas, mesmo, de condições, de falta de medicação, exames..." (APS). “Cinco anos numa fila de espera pra passar num ortopedista." (APS)."[...] aquié uma unidade pequena, pro vasto atendimento que tem aqui, a gente atende é três bairros, três, né? [...] os equipamentos, funcionários não acompanham o crescimento da população..." (APS).

Ainda sobre a organização dos serviços de saúde, foram apreendidas na fala de profissionais e gestores dificuldades relacionadas ao entendimento, por parte dos usuários/ as, sobre o funcionamento da rede de saúde, o que pode contribuir para o percurso pela rede de forma diversa ao estabelecido em normatizações e "[...] é urgência e emergência, mesmo. Chega aqui tudo quanto é coisa que você pode imaginar. Mulher ganhando, quase ganhando menino, braço quebrado, cabeça rachada, mais ou menos assim" (APS). "O nosso funciona como uma central de atendimento, mesmo, não é um PSF" (APS); "[...] foi muito difícil as pessoas entenderem que o seu tratamento poderia continuar com a saúde mental na Atenção Primária, porque elas achavam que se saísse daquele lugar da especialidade, elas não teriam acesso [...]"(AS); "[...] o paciente vem sem, vem sem algumas orientações, às vezes o paciente da unidade básica procura primeiro o hospital, aí a gente tem que encaminhar o paciente" (AT).

Importante destacar a impotência dos profissionais diante das situações vivenciadas e os desafios cotidianos: 
[...] "porque até ele (usuário) chegar nessa referência, ele passou no acolhimento, as filas do acolhimento da odonto é de um ano a dois pra chamar [...] aí quando ele recebe a guia pra atenção secundária, vocêfala assim: 'oh, você esperou um ano e meio, agora, você vai esperar mais um ano"' (AS); "[...] o pessoal faz a contrarreferência pedindo que a unidade tenha um acompanhamento periódico, né, aí só que também as unidades de atenção primária não conseguem muito reabsorver isso, porque não tem vaga" (AS).

Fica claro também que as trajetórias assistenciais dos usuários obedecem a critérios não apenas normativos, como ponderam Viana et al. ${ }^{1: 1795}$ :"[...] os usuários buscam e criam maneiras de acessar os serviços de saúde que desafiam a racionalidade destes mesmos, ou seja, articulam fluxos para sobrevivência ou que respondam a suas demandas reais ou simbólicas". Uma política de territorialização em saúde que supere o entendimento parcial do território pode contribuir para a construção de percursos assistenciais que conectem sentidos (atribuídos por usuários e, também, profissionais) e normatizações (caras à organização dos serviços de saúde). A dimensão"relacional", conforme proposto na análise dos dados, abarca uma série de aspectos subjetivos que perpassam a relação entre usuários e profissionais de saúde.

Os resultados corroboram a perspectiva de que sentidos e atributos caros à APS, como vínculo, confiança e responsabilização entre profissionais e população ${ }^{19,25}$, são mais fortemente desenvolvidos na relação entre usuários e profissionais de saúde inseridos na APS, sobretudo ACS: "Porque, de uma certa forma, a gente cria um vínculo muito grande, porque, querendo ou não, às vezes, com os poucos recursos que a gente tem, a gente quer resolver pra eles, então eu acho que eles sentem isso" (APS).

Como demonstraram os resultados do estudo, essa relação entre usuários e profissionais é influenciada por fragilidades que comprometem o acesso aos serviços de saúde, como o reduzido número de consultas disponíveis para determinadas especialidades médicas e também problemas de entendimento por parte da população usuária sobre o funcionamento da rede de saúde," "[...] tem dias que fica como UPA, sopitando de gente e a gente como profissional, a gente não tem como mandar voltar [...] e eles (usuários) não têm informação" (APS); "Se a necessidade dele for uma demanda muito difícil, tipo uma especialidade, uma ortopedista, o paciente, ele se torna meio que arredio. [...] e aí ele acaba que, às vezes, ele entra em atrito com os funcionários devido a essa dificuldade que a gente tem de marcar a especialidade ou de um exame" (AS).

No entanto, foi possível apreender uma boa relação entre profissionais de saúde e usuários expressa inclusive em relatos sobre o sentimento de impotência de alguns profissionais diante das condições de vida dos usuários: "[...] Se dependesse da gente, funcionava a mil, mas não depende da gente, porque a gente, a gente sente de perto a população, a gente sabe das dificuldades, a gente sabe dos anseios" [...]. "[...] a gente tem bairro aqui que não tem saneamento básico, não tem água, água encanada tem, só não tem esgoto, o esgoto corre a céu aberto, então tudo isso influencia na saúde do paciente" (APS).

Os resultados encontrados corroboram o estudo realizado no município em $2011^{4}$. Guardadas as especificidades relacionadas, sobretudo à abrangência e ao período de estudo, podemos dizer que permanecem os desafios relacionados ao acesso aos serviços de saúde em um território que mantêm elevada vulnerabilidade social e econômica. Os desafios tornamse ainda maiores na configuração política atual de "[...] ofensiva conservadora às políticas sociais, em particular ao SUS, a maior política social que já existiu no país, mesmo com todos os problemas não resolvidos nas suas três décadas de existência" 26:1717.

\section{CONSIDERAÇÕES FINAIS}

A ampliação do estudo realizado em 2011 foi importante para compreender aspectos relacionados ao acesso à rede de atenção à saúde do município. As dimensões teóricas utilizadas contribuíram para análise dos dados e sinalizaram que, de maneira geral, questões estruturais assumiram, na percepção de profissionais e gestores, centralidade como determinantes para o acesso. No estudo de 2011 a dimensão relacional apresentou-se como fundamental 
provavelmente devido à inclusão de usuários, o que não foi possível no presente estudo. Compreendemos, inclusive, esse fato como uma limitação.

A dimensão "estrutural" esteve relacionada não apenas ao financiamento, mas também a questões afetas à operacionalização e organização dos serviços de saúde como rotatividade dos profissionais e planejamento territorial.

Muitas dessas questões não são passíveis de intervenções, pelo menos de forma direta e em curto prazo por profissionais e gestores. No entanto, reconhecer e refletir sobre sua influência no acesso aos serviços da rede de saúde municipal pode ser um produtivo caminho no sentido de articulação, proposição e implementação de estratégias.

\section{REFERÊNCIAS}

1. Viana ALD, Bousquat A, Melo GA, Negri A Fo, Medina MG. Regionalização e Redes de Saúde. Cien Saude Colet. 2018;23(6):1791-8. http://dx.doi.org/10.1590/1413-81232018236.05502018. PMid:29972487.

2. Campos GWS. SUS: o que e como fazer? Cien Saude Colet. 2018;23(60):1707-14. http://dx.doi. org/10.1590/1413-81232018236.05582018. PMid:29972476.

3. Viacava F, Oliveira RAD, Carvalho CC, Laguardia J, Bellido JG. SUS: oferta, acesso e utilização de serviços de saúde nos últimos 30 anos. Cien Saude Colet. 2018;23(6):1751-62. http://dx.doi.org/10.1590/141381232018236.06022018. PMid:29972484.

4. Viegas APB, Carmo RF, Luz ZMP. Fatores que influenciam o acesso aos serviços de saúde na visão de profissionais e usuários de uma unidade de referência. Revista Saúde e Sociedade. 2015;24(1):100-12. http://dx.doi.org/10.1590/S0104-12902015000100008.

5. Travassos C, Castro MSM. Determinantes e desigualdades sociais no acesso e na utilização dos serviços de saúde. In: Giovanella L, organizadora. Políticas e sistema de saúde no Brasil. Rio de Janeiro: Fiocruz/ CEBES; 2008. p. 183-206.

6. Instituto Brasileiro de Geografia e Estatística. Cidades@ [Internet]. Rio de Janeiro: IBGE; 2018 [citado em 2018 jul 12]. Disponível em: https://cidades.ibge.gov.br/brasil/mg/ribeiraodasneves/panorama

7. Instituto de Pesquisa Econômica Aplicada. Atlas da Vulnerabilidade Social (IVS) [Internet]. Brasília: IPEA; 2018 [citado em 2018 jul 7]. Disponível em: http://ivs.ipea.gov.br/index.php/pt/

8. Minayo MCS. O desafio do conhecimento: pesquisa qualitativa em saúde. São Paulo: Hucitec-Abrasco; 2010.

9. Fontanella BJB, Luchesi BM, Saidel MG, Ricas J, Turato ER, Melo DG. Amostragem em pesquisas qualitativas: proposta de procedimentos para constatar saturação teórica. Cad Saude Publica. 2011;27(2):389-94. http://dx.doi.org/10.1590/S0102-311X2011000200020. PMid:21359475.

10. Minayo MCS. Amostragem e saturação em pesquisa qualitativa: consensos e controvérsias. Rev Pesqui Qual. 2017;5(7):1-12.

11. Bardin L. Análise de conteúdo. Lisboa: Edições 70; 2010.

12. Paim JS. Sistema Único de Saúde (SUS) aos 30 anos. Cien Saude Colet. 2018;23(6):1723-8. http://dx.doi. org/10.1590/1413-81232018236.09172018. PMid:29972481.

13. Medeiros CRG, Junqueira AGW, Schwingel G, Carreno I, Jungles LAP, Saldanha OMLF. A rotatividade de enfermeiros e médicos: um impasse na implementação da Estratégia de Saúde da Família. Cien Saude Colet. 2010;15(1 Suppl 1):1521-31. http://dx.doi.org/10.1590/S1413-81232010000700064. PMid:20640314.

14. Machado MH, Ximenes FRG No. Gestão da Educação e do Trabalho em Saúde no SUS: trinta anos de avanços e desafios. Cien Saude Colet. 2018;23(6):1971-9. http://dx.doi.org/10.1590/1413-81232018236.06682018. PMid:29972504.

15. Paim JS. Modelos de Atenção à Saúde no Brasil. In: Giovanella L, Lobato LVC, Noronha JC, Carvalho Al. Políticas e Sistema de Saúde no Brasil. 2. ed. Rio de Janeiro: Fiocruz; 2012. p. 459-491.

16. Brasil. Departamento de Atenção Básica. Portaria n².436, de 21 de setembro de 2017. Aprova a Política Nacional de Atenção Básica, estabelecendo a revisão de diretrizes para a organização da Atenção Básica, no âmbito do Sistema Único de Saúde (SUS). Diário Oficial da União, Brasília, 22 de setembro de 2017; Seção 1.

17. Pereira MPB, Barcellos C. O território no Programa Saúde da Família. Hygeia; Rev Bras Geogr Med Saude. 2006;2(2):47-55. 
18. Solla JJSP, Paim JS. Relações entre a atenção básica, de média e alta complexidade: desafios para a organização do cuidado no Sistema Único de Saúde. In: Paim JS, Almeida-Filho N. Saúde coletiva: teoria e prática. Rio de Janeiro: Editora MedBook; 2014. p. 353-371.

19. Aquino R, Medina MG, Nunes CA, Sousa MF. Estratégia saúde da família e reordenamento do sistema de serviços de saúde. In: Paim JS, Almeida-Filho N. Saúde coletiva: teoria e prática. Rio de Janeiro: Editora MedBook; 2014. p. 343-352.

20. Faria RM, Bortolozzi A. Território e saúde na geografia de Milton Santos: teoria e método para o planejamento territorial do Sistema Único de Saúde no Brasil. Revista Raega - O espaço geográfico em análise. 2016;38:291-320. http://dx.doi.org/10.5380/raega.v38i0.43912.

21. Faria MR. A territorialização da Atenção Primária à Saúde no Sistema Único de Saúde e a construção de uma perspectiva de adequação dos serviços aos perfis do território. Hygeia; Rev Bras Geogr Med Saude. 2013;9(16):131-47.

22. Gondim GMM, Monken M, Rojas LI, Barcellos C, Peiter P, Navarro M, et al. O território da saúde: a organização do sistema de saúde e a territorialização [Internet]. Curitiba: Escola de Saúde Pública do Paraná; 2018 [citado em 2018 ago 23]. Disponível em: http://www.escoladesaude.pr.gov.br/arquivos/ File/TEXTOS_CURSO_VIGILANCIA/20.pdf

23. Santos M. A natureza do espaço. São Paulo: Editora EDUSP; 2014.

24. Santos M. Técnica, espaço, tempo. São Paulo: Editora EDUSP; 2013.

25. Starfield B. Atenção primária de saúde: equilíbrio entre necessidades de saúde, serviços e tecnologia. Brasília: Unesco/Ministério da Saúde; 2002.

26. Rizzotto MLF. A reafirmação da democracia e do direito universal à saúde em tempos de ultraneoliberalismo. Cien Saude Colet. 2018;23(6):1717-8. http://dx.doi.org/10.1590/1413-81232018236.05682018. PMid:29972478. 